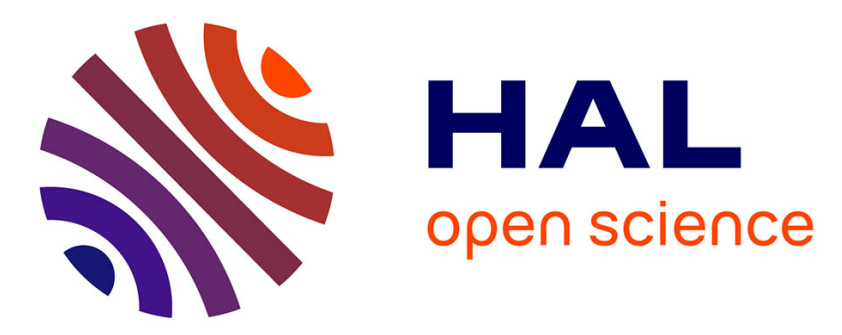

\title{
Multiscale modeling of nonlinear electric conductivity ingraphene-reinforced nanocomposites taking into account tunnelling effect
}

\author{
Xiaoxin Lu, Julien Yvonnet, Fabrice Detrez, Jinbo Bai
}

\section{To cite this version:}

Xiaoxin Lu, Julien Yvonnet, Fabrice Detrez, Jinbo Bai. Multiscale modeling of nonlinear electric conductivity ingraphene-reinforced nanocomposites taking into account tunnelling effect. Journal of Computational Physics, 2017, 337, pp.116-131. 10.1016/j.jcp.2017.01.063 . hal-01463442

HAL Id: hal-01463442

https://hal.science/hal-01463442

Submitted on 9 Feb 2017

HAL is a multi-disciplinary open access archive for the deposit and dissemination of scientific research documents, whether they are published or not. The documents may come from teaching and research institutions in France or abroad, or from public or private research centers.
L'archive ouverte pluridisciplinaire HAL, est destinée au dépôt et à la diffusion de documents scientifiques de niveau recherche, publiés ou non, émanant des établissements d'enseignement et de recherche français ou étrangers, des laboratoires publics ou privés. 


\title{
Multiscale modeling of nonlinear electric conductivity in graphene-reinforced nanocomposites taking into account tunnelling effect
}

\author{
Xiaoxin $\mathrm{Lu}^{\mathrm{a}, \mathrm{b}}$, Julien Yvonnet ${ }^{\mathrm{b}, *}$, Fabrice Detrez ${ }^{\mathrm{b}}$, Jinbo Bai ${ }^{\mathrm{a}}$ \\ ${ }^{a}$ Laboratoire de Mécanique des Sols, Structures et Matériaux, \\ UMR 8579 CNRS, Centrale Supélec, \\ Grande Voie des Vignes, 92290 Châtenay Malabry, France \\ ${ }^{b}$ Université Paris-Est, Laboratoire de Modélisation et Simulation Multi Echelle, \\ UMR 8208 CNRS, \\ 5 Boulevard Descartes, 77454 Marne-la-Vallée Cedex 2, France
}

\begin{abstract}
Tunnelling effect is a possible mechanism to explain the apparent large electric conductivity and nonlinear electric behavior of graphene-reinforced nanocomposites with polymer matrix. In this work, a numerical modeling framework is proposed to evaluate the effective electric conductivity in polymer composites reinforced with graphene sheets, taking into account the electrical tunnelling effect, which allows conduction between graphene sheets at nanometric distances. We introduce a nonlinear Finite Element formulation and a numerical methodology to model the nonlocal and nonlinear effects introduced by the tunnelling effect conduction model within the polymer matrix between close graphene sheets. In addition, to avoid meshing the thickness of the graphene sheets and in view of their very high aspect ratio, a highly conducting surface model is employed. The computed effective conductivity is evaluated over representative volume elements containing arbitrary distributed graphene sheets. The results exhibit tendencies and percolation thresholds which are in qualitative agreement with the available experimental results.
\end{abstract}

Keywords:

graphene/polymer nanocomposites, tunnelling effect, highly conducting

${ }^{*}$ Corresponding author Email: julien.yvonnet@univ-paris-est.fr 
surface model, electric properties

\section{Introduction}

Carbon-based nanofillers (e.g. black carbon, carbon fiber and carbon nanotubes) have been widely used for improving the mechanical, thermal and electric properties of nanocomposites with polymer matrix [1-5]. Graphene, a two dimensional sheet composed of $\mathrm{sp}^{2}$ carbon atoms arranged in a honeycomb structure, has been recently used as filler in polymer-matrix composites for a wide range of applications, due to its giant electrical and mechanical properties [6-10]. Even though polymeric materials are considered as electrical insulators due to their extremely low electrical conductivity, the introduction of graphene can lead to a percolation behavior at very low volume fractions and can increase the conductivity of the resulting composites by several orders of magnitude [11-13]. In recent studies, the percolation threshold for graphene can be as low as $0.07 \%$ in volume fraction due to its extremely high aspect ratio [14].

In the past decade, a number of contributions have been proposed for the simulation of the electric conductivity of nanocomposites with carbonbased filler, mostly with carbon nanotubes (CNTs). A simple model was first set up by Bauhofer and Kovacs [15] to describe the electric conductivity in nanocomposites in the form of a power-law. However, such models are empirical and do not allow sensitivity analysis of the effective electric conductivity of the composite with respect to its microstructural parameters.

Experimental data of the conductivity of composites embedding carbon particles have evidenced two unexpected phenomena: on one hand, the electrical response, i.e. the current-voltage curve can be nonliner for high applied voltages $[16,17]$. On the other hand, the values of the effective conductivity are much higher than expected, considering that the polymer matrix is almost electrically insulating, even when the percolation threshold is reached $[18,19]$. To reproduce these observations, linear models of the conductivity are not sufficient. One possible phenomenon to explain these effects is the electric tunnelling effect [20-22]: for small distances between carbon inclusions (of the order of 1 or $2 \mathrm{~nm}$ ), electrons can cross the energy barrier formed by the polymer layer between both inclusions. In this region, the resulting electric conduction is nonlinear and conduction can be achieved even without perfect contact between the inclusions, resulting in low percolation thresh- 
olds and increased effective conductivity even at very low volume fractions of inclusions [23-25].

$\mathrm{Hu}$ et al [26] and Bao et al [27] considered the tunnelling effect between CNTs and evaluated the effective electric properties of nanocomposites with randomly distributed CNTs. These authors estimated the tunnelling resistance by Simmons' model [28] with the assumption that the electric potential between two tubes is close to zero, and latter introduced the tunnelling channels by Landauer-Büttiker(L-B) model [29] and extended the theory to various types of CNTs. Recently, Wang et al. [30] developed a continuum theory considering the factors that affect the overall conductivity of graphene-based nanocomposites, including the dispersion state of graphene, the imperfectly conducting interface and tunnelling-assisted interfacial conductivity.

To our best knowledge, numerical methodologies involving nonlinear tunnelling effect to evaluate the effective elecric conductivity in composites are quite few. They are however necessary to better understand the local phenomena and the influence of reinforcement parameters on the apparent electric conductivity of the composite and to design materials with higher performances. In the present work, we develop such numerical methodology based on finite elements to study the effective conductivity of graphenepolymer nanocomposites and to predict the percolation thresholds. A FEM formulation involving the nonlinear electric conduction effects is developed on a Representative Volume Element (RVE) containing randomly distributed graphene sheets. To take into account the specific non-localities related to the tunnelling effect, a distance function map is constructed within the RVE model. In addition, an imperfect surface model [31] is introduced to represent the graphene sheets as surface within the RVE model and avoid meshing the thickness of graphene sheets, while incorporating the discontinuities in electric current density in the normal direction of graphene sheets. Finally, a procedure is described to compute the effective electric conductivity of the material from RVE calculations.

The paper is organized as follows. In section 2, the tunnelling effect model, which characterizes the nonlinear conduction mechanisms between close graphene sheets is described. In section 3, the multiscale model of graphene-polymer composite is presented as well as the related homogenization procedure. The FEM discretization of equations to solve the RVE nonlinear problem including the tunnelling effect is described in section 4 with related algorithms. Finally, we apply the present model to analyze the effective nonlinear effective conductivity properties of graphene-polymer composites 
in section 5.

\section{Nonlinear conduction mechanisms in polymer/graphene nanocom- posites: Tunnelling effect}

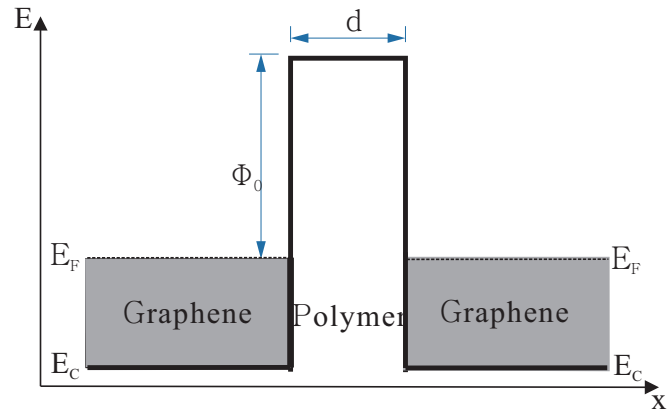

(a)

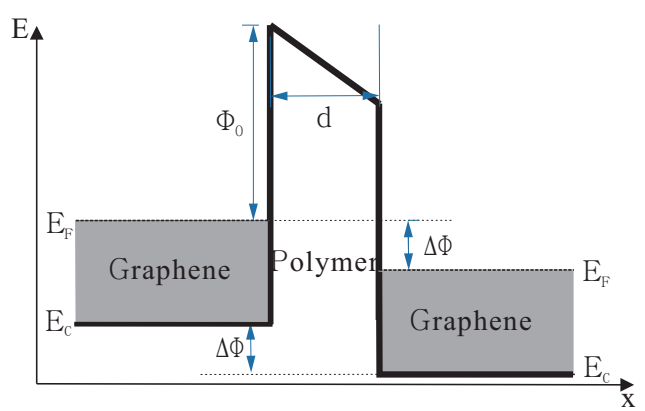

(b)

Figure 1: Band diagrams of a graphene-polymer-graphene unit: (a) at equilibrium $V=0$; (b) with applied voltage $V$. The grey part indicates filling of energy levels, $E_{c}$ is the conduction band edge energy, and $E_{F}$ is the Fermi level in graphene.

As an illustration of this phenomenon, we consider a 1D model of polymer/graphene nanocomposite as a juxtaposition of elementary graphenepolymer-graphene units (see Figure 1 (a)). In order to understand the nonlinear electrical behavior of the nanocomposite, we first focus on the conduction mechanism of this unit. When the distance between two graphene sheets is nano-scaled, the polymer matrix can be seen as a dielectric film between two graphene electrodes. There is a strong analogy with a metal-insulatormetal device with tunnelling junction. It is known that there are two types of conduction mechanism in dielectric films [32], which are interface-limited conduction mechanism and bulk-limited conduction mechanism respectively. The former depends on the electric properties at the polymer-graphene interface, while the latter depends on the electric properties of the polymer. In our work, we focus on solving the general problem and neglect the bulk-limited conduction mechanism except Ohm's law in varying polymer.

There are several interface-limited conduction mechanisms [33]: (1) Schottky emission; (2) Thermionic-field emission; (3) Fowler-Nordheim tunnelling; (4) Direct tunnelling. Among them, the former two are significant only at high temperature when the electrons can get enough thermal energy to overcome the barrier so that the electronic current can flow in the conduction 
band. The latter two occur when the barrier is thin enough to permit its penetration by the quantum tunnelling [34]. To simplify the problem, we assume that the system is studied at low temperature so that the thermal current can be neglected, and restrict the electron transportation between electrodes to the tunnelling effect.

The tunnelling effect is a purely quantum effect. For a 1D model of two close graphene sheets separated by a polymer matrix, the two graphene sheets are considered as source of electrons, and the polymer layer as a potential barrier. Figure 1(a) depicts the evolution of conduction band edge energy, $E_{c}$ at equilibrium state across the graphene-polymer-graphene device. The potentiel barrier in polymer corresponding to energy level of conduction band edge is assumed to have a rectangular shape at equilibrium, e.i. the interface effect is neglected in first approximation. When the electrostatic potential $V$ changes, the conduction band edge energy is shifted by $\Delta \phi=-e V$, where $e$ is the elementary electric charge, that is why the barrier shape becomes trapezoidal when the graphene have different electrostatic potential.

For such 1D model of two close graphene sheets separated by a polymer matrix, the electric constitutive law is expressed in the polymer in the form

$$
j=\mathcal{G}(E, d) E
$$

where $j$ denotes the electric current, $E$ is the electric field, and $\mathcal{G}(E, d)$ is a nonlinear function of the electric field $E$ and of the distance between the two graphene sheets $d$. An explicit formula for the electric tunnelling effect through a potential square barrier was first derived by Simmons [28] as:

$$
\begin{aligned}
\mathcal{G}(E, d)= & \frac{2.2 e^{3} E^{2}}{8 \pi h \Phi_{0}} \exp \left(-\frac{8 \pi}{2.96 h e E}(2 m)^{\frac{1}{2}} \Phi_{0}^{\frac{3}{2}}\right) \ldots \\
& +\left[3 \cdot \frac{\left(2 m \Phi_{0}\right)^{\frac{1}{2}}}{2 d}\right](e / h)^{2} E d \exp \left[-\left(\frac{4 \pi d}{h}\right)\left(2 m \Phi_{0}\right)^{\frac{1}{2}}\right] .
\end{aligned}
$$

Here, the tunnelling current $j$ is a nonlinear function of the electric field $E$ and depends of three parameters: the height $\Phi_{0}$, width $d$ of barrier and the effective mass $m$ of electron in graphene. This expression is obtained under the assumptions that the temperature is very low such as $T \approx 0 \mathrm{~K}$; the electric field $E$ varies slowly over the width of the potential barrier (i.e. $E \approx V / d$ and the rectangular barrier becomes trapezoidal under the effect of electric field (see. Fig. 1b)); the trapezoidal barrier is approximated by rectangular barrier with the same average height. This theory has been 


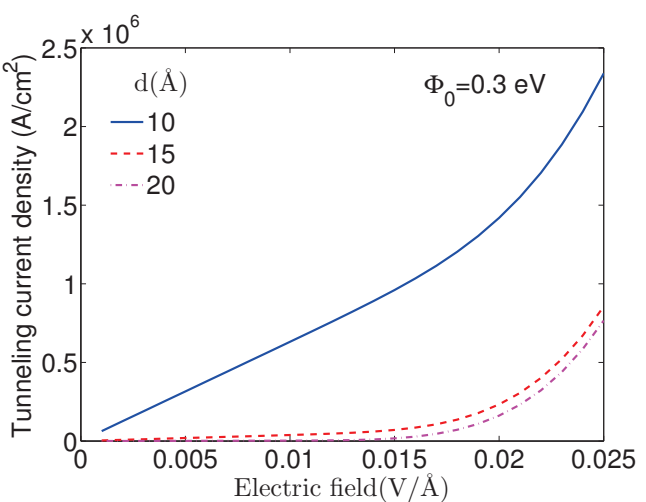

(a)

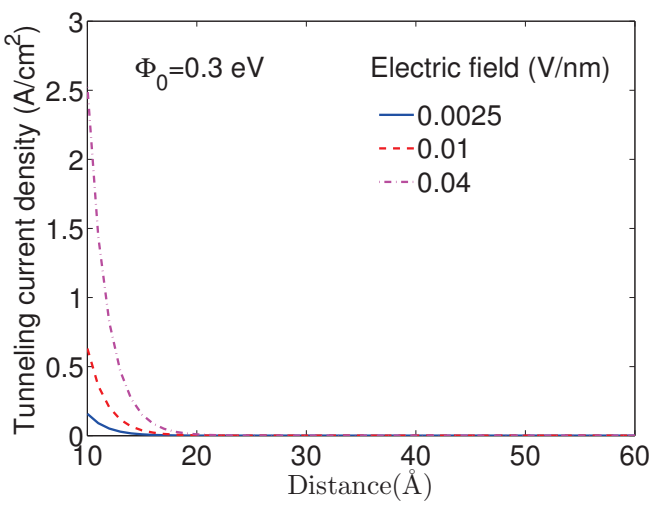

(b)

Figure 2: Characteristic behaviors of a tunnelling junction with rectangular barrier: (a) tunnelling current density versus Electric field; (b) tunnelling current density versus barrier width.

used and validated experimentally in the metal-insulator-semiconductivity [35] and metal-insulator-metal capacitor field [36]. Note that the current comes only from the electrons which cross the potential barrier. In Simmons' model the leakage current is not taken into account and is thus not modeled in the present work.

Figure 2 shows the evolution of the tunnelling current as a function of $E$ and $d$ respectively. Specifically, when $d$ is a constant, the tunnelling current increases with electric field $E$ (see Fig. 2 (a)), while with fixed electric field, an increase of $d$ leads to a sharp decrease of tunnelling current (see in Fig. 2 (b)).

\section{Multiscale Modeling of the electrical behavior of graphene-reinforced composites}

We consider an RVE defined in a domain $\Omega$ whose external boundary is denoted by $\partial \Omega$. The RVE contains $N$ planar graphene sheets associated to surfaces $\Gamma_{n}, n=1,2, \ldots, N$, which are distributed randomly inside the polymer matrix, as depicted in Fig. 3. The distribution of graphene sheets is assumed to be periodic. We denote collectively the graphene surfaces by $\Gamma=\bigcup_{n} \Gamma_{n}$. The homogenization problem defined over the RVE $\Omega$ is described in the following. 


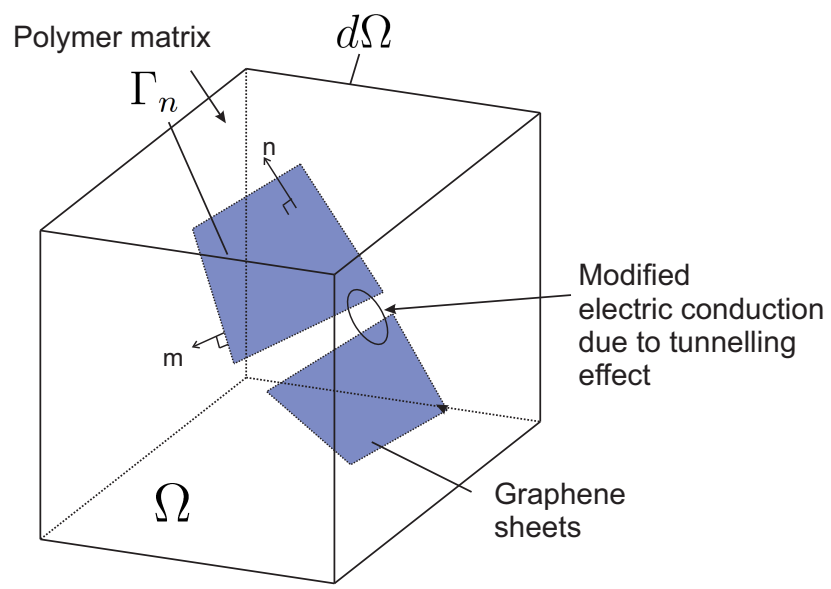

Figure 3: RVE model of the graphene-reinforced composite.

\subsection{Microscopic problem}

Considering only electrical conduction phenomena, the electric power within the domain $\Omega$ is defined by

$$
\bar{W}=\int_{\Omega} \omega^{b}(\mathbf{x}) d \Omega+\int_{\Gamma} \omega^{s}(\mathbf{x}) d \Gamma,
$$

where the density functions $\omega^{b}$ and $\omega^{s}$ are defined by

$$
\omega^{b}(\mathbf{x})=\mathbf{j}(\mathbf{x}) \cdot \mathbf{E}(\mathbf{x}), \quad \omega^{s}(\mathbf{x})=\mathbf{j}^{s}(\mathbf{x}) \cdot \mathbf{E}^{s}(\mathbf{x}),
$$

where

$$
\mathbf{E}(\mathbf{x})=-\nabla \phi(\mathbf{x})
$$

is the electric field, $\mathbf{j}(\mathbf{x})$ is the current density vector and $\phi(\mathbf{x})$ is the electric potential. In the above, the superscript $s$ denotes surface quantities, e.g., $\mathbf{j}^{s}$ is the surface current density. The surface electric field is defined with respect to its bulk counterpart as:

$$
\mathbf{E}^{s}=\mathbf{P E}=-\nabla^{s} \phi=-\mathbf{P} \nabla \phi
$$

with

$$
\mathbf{P}(\mathbf{x})=\mathbf{1}-\mathbf{n}(\mathbf{x}) \otimes \mathbf{n}(\mathbf{x})
$$

is a projector operator characterizing the projection of a vector along the tangent plane to $\Gamma$ at a point $\mathbf{x} \in \Gamma$ and $\mathbf{n}$ is the unit normal vector to $\Gamma$. 
The local constitutive relationships relating $\mathbf{j}$ and $\mathbf{j}^{s}$ with $\mathbf{E}$ are introduced in section 3.2. In (3) the electric potential is assumed to verify the condition

$$
\langle-\nabla \phi(\mathbf{x})\rangle=\overline{\mathbf{E}}
$$

where $\langle\rangle=.\frac{1}{V} \int_{\Omega}(). d \Omega$ is the spatial averaging over $\Omega$, with $V$ the volume of $\Omega$. This condition is verified for the following boundary conditions over $\partial \Omega$ :

$$
\begin{array}{lr}
\phi(\mathbf{x})=-\overline{\mathbf{E}} \cdot \mathbf{x} & \text { on } \partial \Omega \\
\phi(\mathbf{x})=-\overline{\mathbf{E}} \cdot \mathbf{x}+\tilde{\phi}(\mathbf{x}) & \text { on } \partial \Omega
\end{array}
$$

where $\tilde{\phi}(\mathbf{x})$ is a periodic function over $\Omega$, such as $\langle\tilde{\phi}(\mathbf{x})\rangle=0$. In the present paper, the second type of boundary conditions has been adopted.

\subsection{Local microscopic constitutive equations}

\subsubsection{Polymer matrix}

In the polymer matrix, the current density $\mathbf{j}$ is related to the electric field through the nonlinear relationship:

$$
\mathbf{j}= \begin{cases}\mathbf{k}_{p}^{0} \mathbf{E} & \text { if } d(\mathbf{x})>d_{c u t}, \\ \mathcal{G}(\mathbf{E}, d) \frac{\mathbf{E}}{|\mathbf{E}|} & \text { if } d(\mathbf{x})<d_{c u t},\end{cases}
$$

where $d_{c u t}$ is a cut-off distance above which the tunnelling effect can be neglected, $\mathbf{k}_{p}^{0}$ is the electric conductivity tensor of the polymer matrix when neglecting tunnelling effect and $\mathcal{G}$ is defined by (2). Here, we make the assumption that the tunneling effect is independent of the graphene thickness.

\subsubsection{Graphene sheets}

Graphene sheets are composed of several layers of graphene and thus have a finite thickness denoted by $h$. However, due to the very large aspect ratio of graphene sheets, which can be of the the order of $10^{3}$ between the largest dimension of the sheet and the thickness, it might be cumbersome to model them as volume domains, especially regarding meshing the thickness of the sheets. For this reason, we propose to replace the graphene sheets with finite thickness by highly conducting surfaces (see Fig. 4). In that case, the graphene sheets defined in a volume domain $\Omega^{g}$ are modeled by imperfect surfaces $\Gamma$ where the surface current density $\mathbf{j}^{s}$ is in that case related to the surface electric field, $\mathbf{E}^{s}$ through (see e.g. [31, 37]): 


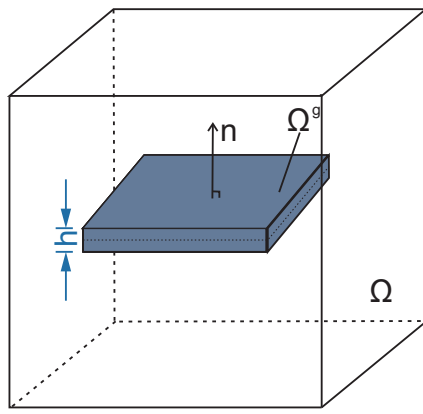

(a)

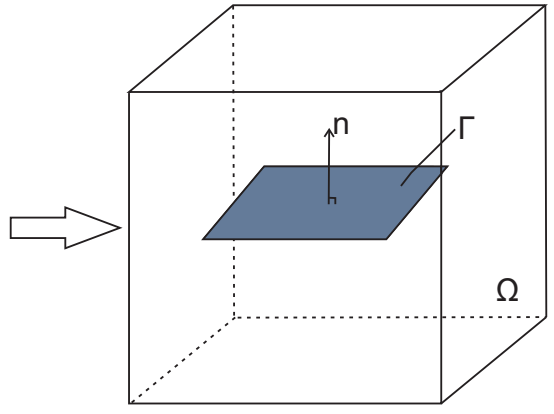

(b)

Figure 4: Replacement of 3D graphene by a highly conducting surface.

$$
\mathbf{j}^{s}(\mathbf{x})=\mathbf{k}^{s} \mathbf{E}^{s}
$$

where

$$
\mathbf{k}^{s}=h \mathbf{S}, \quad \mathbf{S}=\mathbf{k}_{g}-\frac{\left(\mathbf{k}_{g} \mathbf{n}\right) \otimes\left(\mathbf{k}_{g} \mathbf{n}\right)}{\mathbf{k}_{g}:(\mathbf{n} \otimes \mathbf{n})} .
$$

In (13), $\mathbf{k}^{g}$ denotes the second-order electric conductivity tensor of the bulk graphite, which is here anisotropic.

\subsection{Homogenized quantities}

In this section, we define the effective quantities and their relation to microscopic (local) fields.

\subsubsection{Effective electric displacement}

From energy consistency, we have:

$$
\bar{W}=V \bar{J} \cdot \overline{\mathbf{E}},
$$

where $V$ is the volume of $\Omega$,

$$
\overline{\mathbf{E}}=\frac{1}{V} \int_{\Omega} E(\mathbf{x}) d \Omega
$$

is the effective electric field and $\overline{\mathbf{J}}$ is the effective current density defined by

$$
\overline{\mathbf{J}}=\frac{1}{V} \frac{\partial \bar{W}}{\partial \overline{\mathbf{E}}}
$$


Using (3), we have:

$$
\begin{aligned}
\bar{J}_{i} & =\frac{1}{V} \int_{\Omega} \frac{\partial \omega^{b}}{\partial \bar{E}_{i}} d \Omega+\frac{1}{V} \int_{\Gamma} \frac{\partial \omega^{s}}{\partial \bar{E}_{i}} d \Gamma \\
& =\frac{1}{V} \int_{\Omega} \frac{\partial \omega^{b}}{\partial E_{j}} \frac{\partial E_{j}}{\partial \bar{E}_{i}} d \Omega+\frac{1}{V} \int_{\Gamma} \frac{\partial \omega^{s}}{\partial E_{j}^{s}} \frac{\partial E_{j}^{s}}{\partial \bar{E}_{i}} d \Gamma .
\end{aligned}
$$

Using equation (15), we obtain:

$$
\frac{\partial \bar{E}_{i}}{\partial E_{j}}=\frac{1}{V} \int_{\Omega} \delta_{i j} d \Omega=\delta_{i j}
$$

and

$$
\frac{\partial \omega^{s}}{\partial E_{j}^{s}} \frac{\partial E_{j}^{s}}{\partial \bar{E}_{i}}=j_{j}^{s} P_{i j}=j_{i}^{s} .
$$

Thus, we finally obtain using (4):

$$
\overline{\mathbf{J}}=\frac{1}{V}\left(\int_{\Omega} \mathbf{j}(\mathbf{x}) d \Omega+\int_{\Gamma} \mathbf{j}^{s}(\mathbf{x}) d \Gamma\right) .
$$

\subsubsection{Effective behavior}

Due to the nonlinear term (11), classical homogenization principles related to the superposition principle do not hold. Nonlinear homogenization problems can be handled e.g. by numerical approaches where problems at both scales are concurrently solved ( $\mathrm{FE}^{2}$-approaches), see reviews e.g. in $[38,39]$. In the present case, we restrict the analysis to evaluate the tangent (incremental) effective conductivity tensor, which is defined as:

$$
\overline{\mathbf{k}}_{T}(\overline{\mathbf{E}})=\frac{\partial \overline{\mathbf{J}}(\overline{\mathbf{E}})}{\partial \overline{\mathbf{E}}}
$$

where $\overline{\mathbf{k}}_{T}(\overline{\mathbf{E}})$ is here evaluated numerically by perturbation.

\section{Finite element numerical solving procedure}

\subsection{Weak forms}

The solution $\phi(\mathbf{x})$ satisfying the periodical boundary conditions in Eq. (10) and minimizing the energy verifies:

$$
D_{\delta \phi} \bar{W}(\phi)=0
$$


where $D_{\delta \phi} \bar{W}(\phi)$ denotes the directional (Gâteaux) derivative defined as:

$$
D_{\delta \phi} \bar{W}(\phi)=\left\{\frac{d}{d \epsilon}[\bar{W}(\phi+\epsilon \delta \phi)]\right\}_{\epsilon=0} .
$$

From (22) and using (3) we obtain:

$$
\int_{\Omega} \frac{\partial \omega^{b}}{\partial \mathbf{E}} \cdot D_{\delta \phi}(\mathbf{E}) d \Omega+\int_{\Gamma} \frac{\partial \omega^{s}}{\partial \mathbf{E}^{s}} \cdot D_{\delta \phi}\left(\mathbf{E}^{s}\right) d \Gamma=0,
$$

where

$$
D_{\delta \phi}(\mathbf{E})=-\nabla(\delta \phi)
$$

and

$$
D_{\delta \phi}\left(\mathbf{E}^{s}\right)=-\mathbf{P} \nabla^{s}(\delta \phi)=-\nabla^{s}(\delta \phi) .
$$

Using (4), we obtain the weak form:

$$
\int_{\Omega} \mathbf{j}(\phi) \cdot \nabla(\delta \phi) d \Omega+\int_{\Gamma} \mathbf{j}^{s} \cdot \nabla^{s}(\delta \phi) d \Gamma=R=0,
$$

where $\phi \in H^{1}(\Omega)$, $\phi$ satisfying the boundary conditions (10) over $\partial \Omega$ and $\delta \phi \in H^{1}(\Omega), \delta \phi=0$ over $\partial \Omega$. Introducing expressions (6) and (12) in equation (27) yields:

$$
\int_{\Omega} \mathbf{j}(\phi) \cdot \nabla(\delta \phi) d \Omega-\int_{\Gamma} \mathbf{P} \nabla \phi \cdot \mathbf{k}^{s} \mathbf{P} \nabla(\delta \phi) d \Gamma=R=0 .
$$

\subsection{Linearization}

Due to the term $\mathbf{j}(\phi)$ expressed by (11), the weak form (28) constitutes a highly nonlinear problem with respect to $\phi$. To solve this problem, we employ a Newton-Raphson procedure. For this purpose, the linearization of (28) is provided in what follows.

A first-order Taylor expansion of $R$ gives:

$$
R\left(\phi_{k}+\Delta \phi_{k}\right) \simeq R\left(\phi_{k}\right)+D_{\Delta \phi} R\left(\phi_{k}\right) .
$$

In the above, $\phi_{k}$ denotes the electric potential solution field known from a previous iteration $k, \Delta \phi_{k}$ is the increment of electric potential. Equating the left-hand term of (29) to zero gives the linearized problem around the know solution $\phi_{k}$ as:

$$
D_{\Delta \phi} R\left(\phi_{k}\right)=-R\left(\phi_{k}\right) .
$$


The left-hand term in (30) can be expressed by:

$$
\int_{\Omega} \frac{\partial \mathbf{j}}{\partial \mathbf{E}} \nabla(\Delta \phi) \cdot \nabla(\delta \phi) d \Omega-\int_{\Gamma} \mathbf{P} \nabla(\Delta \phi) \cdot \mathbf{k}^{s} \mathbf{P} \nabla(\delta \phi) d \Gamma,
$$

where

$$
\frac{\partial \mathbf{j}}{\partial \mathbf{E}}=\mathbf{k}_{p}(\mathbf{E})= \begin{cases}\mathbf{k}_{p}^{0} & \text { if } d(\mathbf{x})>d_{c u t} \\ \mathcal{G}^{\prime}(\|\mathbf{E}\|) \cdot \frac{\mathbf{E} \otimes \mathbf{E}}{\|\mathbf{E}\|^{2}}+\mathcal{G}(\|\mathbf{E}\|) \cdot \frac{\|\mathbf{E}\|^{2} \mathbf{I}-\mathbf{E} \otimes \mathbf{E}}{\|\mathbf{E}\|^{3}} & \text { if } d(\mathbf{x}) \leq d_{c u t}\end{cases}
$$

where $\mathcal{G}^{\prime}(|\mathbf{E}| \mid)$ is expressed by:

$$
\mathcal{G}^{\prime}(\|\mathbf{E}\|)=2 A\|\mathbf{E}\| \exp \left(-\frac{B}{\|\mathbf{E}\|}\right)+A B \exp \left(-\frac{B}{\|\mathbf{E}\|}\right)+C,
$$

with

$$
A=\frac{2.2 e^{3}}{8 \pi h \Phi_{0}}, \quad B=\frac{8 \pi}{2.96 h e}(2 m)^{\frac{1}{2}} \Phi_{0}^{\frac{3}{2}}
$$

and

$$
C=3 \cdot \frac{\left(2 m \Phi_{0}\right)^{\frac{1}{2}}}{2}(e / h)^{2} \exp \left[-\left(\frac{4 \pi d}{h}\right)\left(2 m \Phi_{0}\right)^{\frac{1}{2}}\right] .
$$

\subsection{Nonlinear finite element discretization and approximation}

The approximation of the electric potential at a point $\mathbf{x}$ in a volume element $\Omega^{e}$ is expressed as

$$
\phi(\mathbf{x})=\sum_{i=1}^{n} \mathbf{N}^{e} \mathbf{\Phi}^{e}
$$

where $\mathbf{N}^{e}$ is a line vector of shape functions and $\boldsymbol{\Phi}^{e}$ is a column vector containing the nodal values of the electric field for one element. We introduce the following classical FEM discretizations:

$$
\nabla\left(\Phi_{k}^{e}\right)=\mathbf{B}^{e} \boldsymbol{\Phi}_{k}^{e}, \quad \nabla\left(\Delta \Phi_{k}^{e}\right)=\mathbf{B}^{e} \Delta \boldsymbol{\Phi}_{k}^{e}, \quad \nabla(\delta \Phi)=\mathbf{B}^{e} \delta \boldsymbol{\Phi}^{e},
$$

where $\mathbf{B}^{e}$ denotes a matrix of shape functions derivatives. Introducing the above discretization in (30) gives:

$$
\begin{array}{r}
\delta \boldsymbol{\Phi}^{e T}\left(\int_{\Omega} \mathbf{B}^{e T} \mathbf{k}_{T}^{T}\left(\phi_{k}\right) \mathbf{B}^{e} d \Omega\right) \Delta \boldsymbol{\Phi}_{k}^{e}+\delta \boldsymbol{\Phi}^{e T}\left(\int_{\Gamma} \mathbf{B}^{e T} \mathbf{P k}^{s} \mathbf{P} \mathbf{B}^{e} d \Gamma\right) \Delta \boldsymbol{\Phi}_{k}^{e} \\
=-\delta \boldsymbol{\Phi}^{e T}\left(\int_{\Omega} \mathbf{B}^{e T} \mathbf{j}\left(\phi_{k}\right) d \Omega\right)-\delta \boldsymbol{\Phi}^{e T}\left(\int_{\Gamma} \mathbf{B}^{e T} \mathbf{P} \mathbf{k}^{s} \mathbf{P} \mathbf{B}^{e} \boldsymbol{\Phi}_{k}^{e} d \Gamma\right) .
\end{array}
$$


Owing to the arbitrariness of $\delta \boldsymbol{\Phi}^{e}$, we obtain the following system of linear equations:

$$
\left(\mathbf{K}^{b}+\mathbf{K}^{s}\right) \Delta \boldsymbol{\Phi}_{k}=-\mathbf{R}^{b}-\mathbf{R}^{s},
$$

where $\mathbf{K}^{b}$ and $\mathbf{K}^{s}$ denote the bulk and surface tangent matrices, $\mathbf{R}^{b}$ and $\mathbf{R}^{s}$ are residual terms and $\Delta \boldsymbol{\Phi}_{k}$ is the column vector of potential increments for the whole mesh. The different above matrices and vectors are given by:

$$
\begin{gathered}
\mathbf{K}^{b}=\int_{\Omega} \mathbf{B}^{e T} \mathbf{k}^{p}\left(\mathbf{E}\left(\phi_{k}\right)\right) \mathbf{B}^{e} \mathbf{d} \Omega, \quad \mathbf{K}^{s}=\int_{\Gamma^{e}} \mathbf{B}^{e T} \mathbf{P k}^{\mathbf{s}} \mathbf{P} \mathbf{B}^{e} d \Gamma, \\
\mathbf{R}^{b}=\int_{\Omega^{e}} \mathbf{B}^{e T} \mathbf{j}\left(\phi_{k}\right) \mathbf{d} \Omega, \quad \mathbf{R}^{s}=\int_{\Gamma^{e}} \mathbf{B}^{e T} \mathbf{P} \mathbf{k}^{s} \mathbf{P} \mathbf{B}^{e} \boldsymbol{\Phi}_{k}^{e} \mathbf{d} \Gamma .
\end{gathered}
$$

where $\mathbf{k}^{p}\left(\mathbf{E}\left(\phi^{k}\right)\right)$ is given by (32). At each iteration, the electric potential field is udpated according to:

$$
\boldsymbol{\Phi}_{k+1}=\boldsymbol{\Phi}_{k}+\Delta \boldsymbol{\Phi}_{k} .
$$

The process is repeated until the criterion $\|\mathbf{R}\| \leqslant 10^{-6}$ is reached.

One specificity of the present problem is the presence of the surface electrical energy term in Eq. (28) related to the graphene surface. To evaluate the associated surface integral, we first mesh the graphene surface by triangular elements, which then conform with the tetrahedral elements in the volume. Here, a special attention must be paid to the computation of the surface integrals in (40) and (41). The integrals are evaluated with Gauss points positioned along the graphene surface, then on the centers of triangular elements meshing the surface. It is worth noting that the shape functions $\mathbf{B}^{e}$ are discontinuous along $\Gamma$ but that from (27), the quantity $\mathbf{B}^{e T}(\mathbf{x}) \mathbf{P}(\mathbf{x}) \mathbf{k}^{s} \mathbf{P}(\mathbf{x}) \mathbf{B}^{e}(\mathbf{x})$ is continuous across $\Gamma$. Then, the values of $\mathbf{B}^{e}(\mathbf{x})$ being constant in each linear element, they can be evaluated in a Gauss point $\mathrm{x}^{\prime}$ located within neighbor elements, as depicted in Fig. 5, according to:

$$
\mathbf{B}^{T}(\mathbf{x}) \mathbf{P}(\mathbf{x}) \mathbf{k}^{s} \mathbf{P}(\mathbf{x}) \mathbf{B}(\mathbf{x}) \simeq \mathbf{B}^{T}\left(\mathbf{x}^{\prime}\right) \mathbf{P}(\mathbf{x}) \mathbf{k}^{s} \mathbf{P}(\mathbf{x}) \mathbf{B}\left(\mathbf{x}^{\prime}\right) .
$$

The microstructure of the composites is composed of randomly distributed graphene sheets within the polymer matrix. We model graphene sheets as square surfaces of length $L$. Graphene sheets are allowed to cross the boundary, but the periodicity is respected. Graphene sheets cannot cut each others. We used GMSH mesh generator [40] to create the triangular mesh of surfaces conforming with matrix tetrahedra. 


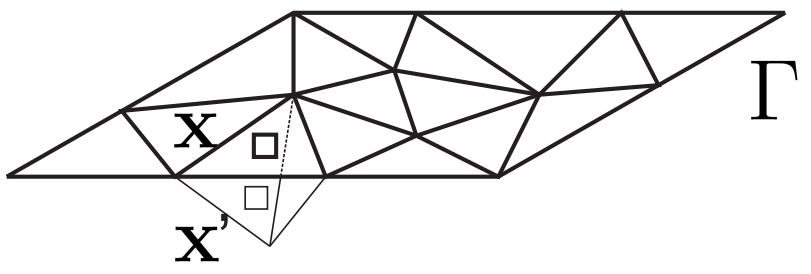

Figure 5: Schematic of the mesh for a graphene sheet and one neighbor element for the computation of integral terms.

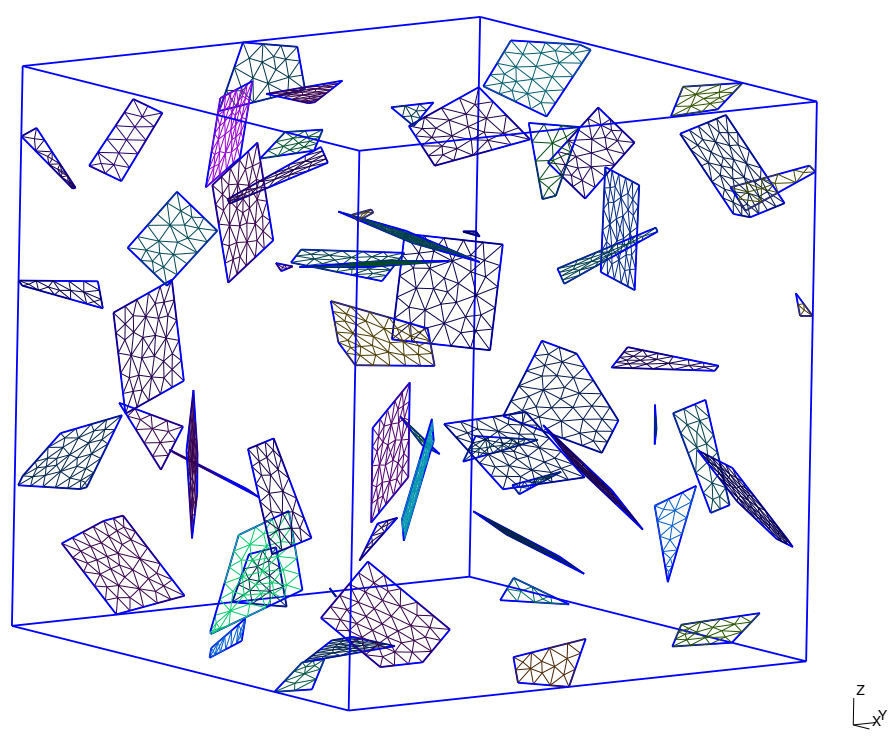

Figure 6: RVE for the graphene/polymer nanocomposites involving 30 graphene sheets in a cube of $80 \times 80 \times 80 \mathrm{~nm}^{3}$. Some graphene sheets seem to have a polygonal shape as they intersect the RVE boundaries. However, the microstructure periodicity ensures that all graphene sheets are of square shape. 


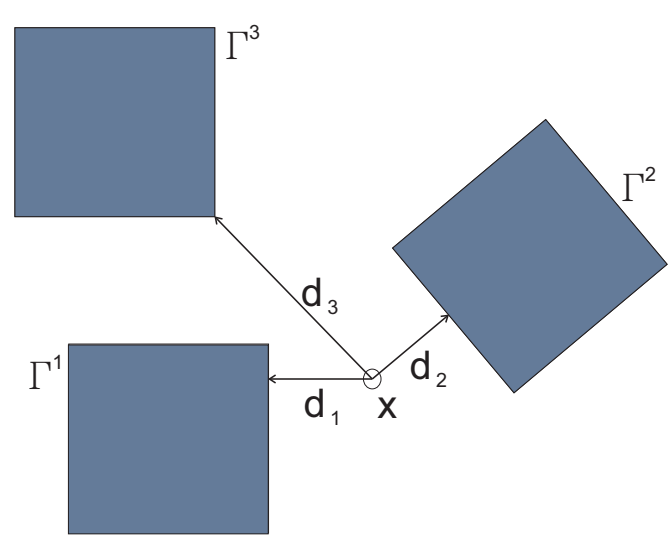

(a)

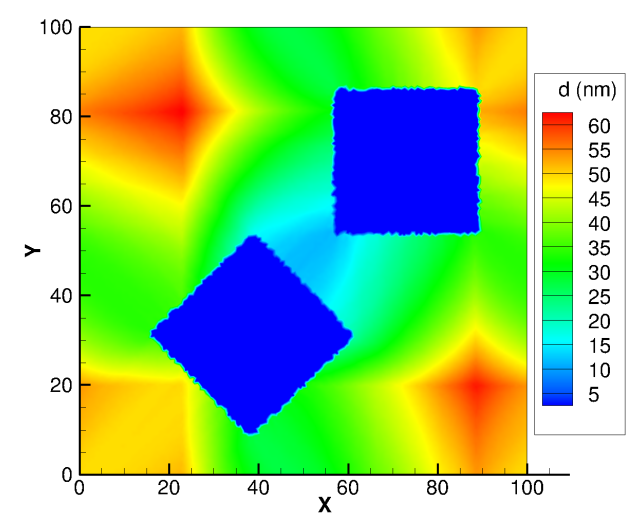

(b)

Figure 7: a) Distances of a point $\mathbf{x}$ from surrounding graphene sheets to compute the distance $d(\mathbf{x})$. b) Distance function $d(\mathbf{x})$ in 2D RVE with peridodic boundary conditions.

\section{4. $3 D$ distance function used in the tunnelling effect model}

We have introduced in section 2 a 3D model where the electric conduction is dependent on a distance $d$ between the graphene sheets. Even though this notion is trivial in $1 \mathrm{D}$, many possible choices can be proposed in a full 3D context. In what follows, we propose a simple definition for the distance function $d(\mathbf{x})$ in equation (11), which can be computed at all nodes of the mesh once before the calculations for a given distribution of graphene sheets within the RVE. Note that this definition is a possible choice among many others and is part of the model. We consider a point $\mathbf{x} \in \Omega$ and denote by $\mathbf{x}^{\Gamma}$ a point lying on the surface $\Gamma$ formed by the set of $N$ graphene sheets. We define the distance function $d(\mathbf{x})$ as follows:

$$
d(\mathbf{x})=\min _{\substack{\mathbf{x}^{\Gamma} \in \Gamma^{i} \\ i=1,2, \ldots, N}}\left\|\mathbf{x}-\mathbf{x}^{\Gamma}\right\|+\min _{\substack{\mathbf{x}^{\Gamma} \in \Gamma^{j} \\ j=1,2, \ldots, N, \quad j \neq i}}\left\|\mathbf{x}-\mathbf{x}^{\Gamma}\right\| .
$$

In other words, for a given point $\mathbf{x}$, we first compute the distances with all $N$ graphene sheets, then the function $d(\mathbf{x})$ is defined as the sum of the two smallest distances between the point and two different neighboring graphene sheets. An illustration of this methodology is schematically depicted in Fig. $7(\mathrm{a})$ in the $2 \mathrm{D}$ context. For one point $\mathbf{x}$, the distances $d_{1}, d_{2}$ and $d_{3}$ represent the shortest distance with graphene sheets $\Gamma^{1}, \Gamma^{2}$ and $\Gamma^{3}$. The function $d(\mathbf{x})$ 


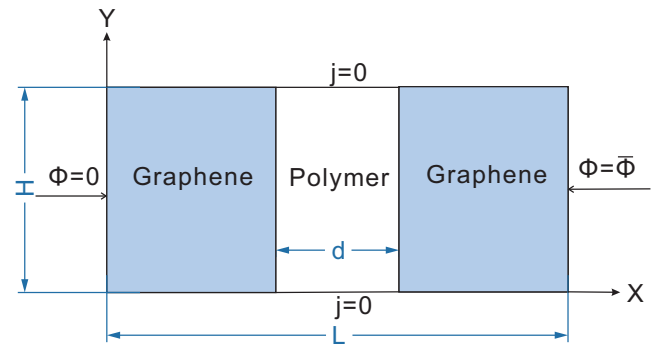

(a)

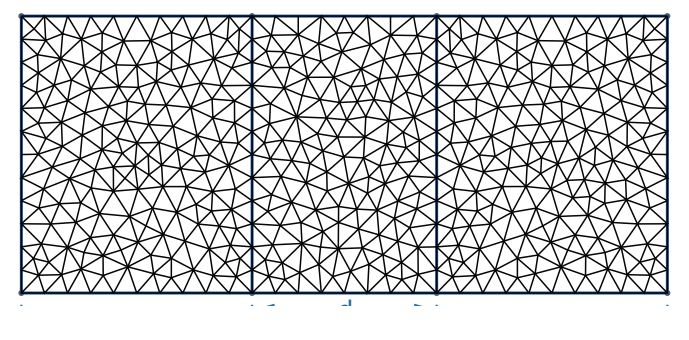

(b)

Figure 8: Benchmark problem to validate the 1D model: (a) geometry and boundary conditions; (b) mesh of the 2D domain.

for this point is the sum of $d_{1}$ and $d_{2}$, which are the smallest values in the set $d_{i}, i=1,2,3$. An illustration of the obtained function $d(\mathbf{x})$ for the $2 \mathrm{D}$ geometry is shown in Fig. 7(b).

\section{Numerical examples}

\section{1. $1 D$ benchmark example}

The objective of this first example is to validate the present model on a simple benchmark where an analytical solution can be obtained, and where the tunnelling effect can be evidenced. The problem is defined in Fig. 8(a). A domain is composed of two regions associated with graphene and a third one associated with the polymer. The width of the polymer layer (distance between the graphene sheets) is denoted by $d$. The length of the domain is $L=d+2 W$, where $W$ denotes the width of graphene sheets, here chosen as $W=10 \AA$. Electric potentials $\phi=0$ and $\phi=\bar{\phi}$ are prescribed at $(x=0)$ and $(x=L)$. Whereas the problem is purely $1 \mathrm{D}$, we solve it in a $2 \mathrm{D}$ domain meshed with linear triangular elements as shown in Fig. 8(b). To avoid any influence of the $y$-coordinate and maintain the problem one-dimensional, zero normal electric current density is prescribed at $(y=0)$ and $(y=H)$

In all the following examples, the electric conductivity of the polymer is taken as $k_{p}^{0}=1 \times 10^{-10} \mathrm{~S} / \mathrm{m}$ and the conductivity tensor is

$$
\mathbf{k}_{p}=\left[\begin{array}{ccc}
1 \times 10^{-10} & 0 & 0 \\
0 & 1 \times 10^{-10} & 0 \\
0 & 0 & 1 \times 10^{-10}
\end{array}\right] \mathrm{S} / \mathrm{m} .
$$




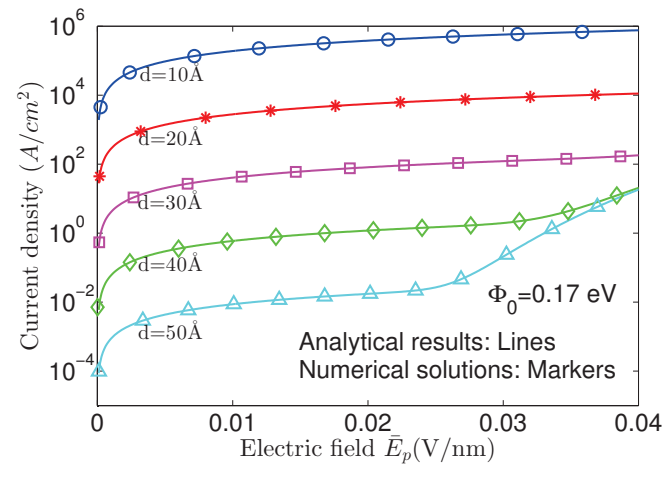

(a)

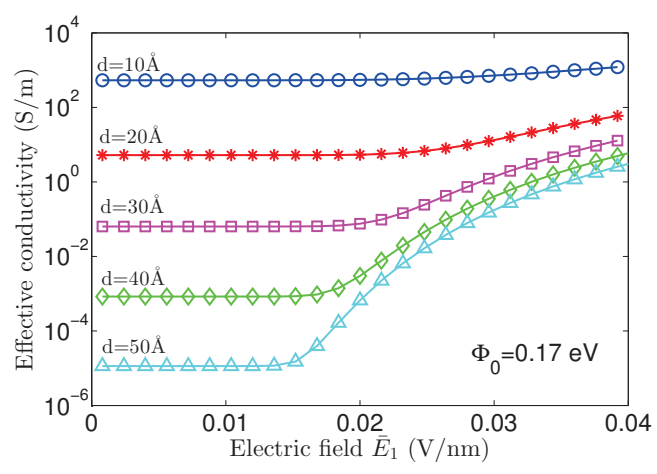

(b)

Figure 9: (a) Effective current density as a function of $\bar{E}_{p}$; (b) Effective electric conductivity as a function of $\bar{E}_{1}$ at different barrier width.

Note that in this benchmark example, the conductivity in the direction normal to the graphene sheet is not taken into account. The conductivity of graphene is anisotropic and its in-plane conductivity was adopted from Stankovich et al. [41], who gave a range of $10^{4.92 \pm 0.52} \mathrm{~S} / \mathrm{m}$. We then used the value of $10^{4.92} \mathrm{~S} / \mathrm{m}$ and therefore take the in-plane conductivity $\left(k_{g}\right)_{1}$ $=8.32 \times 10^{4} \mathrm{~S} / \mathrm{m}$. The out-plane conductivity of the graphene is taken as $\left(k_{g}\right)_{3}=10^{-3}\left(k_{g}\right)_{1}$, or:

$$
\mathbf{k}_{g}=\left[\begin{array}{ccc}
8.32 \times 10^{4} & 0 & 0 \\
0 & 8.32 \times 10^{4} & 0 \\
0 & 0 & 83.2
\end{array}\right] \mathrm{S} / \mathrm{m}, \text { for } 3 \mathrm{D} \text { examples. }
$$

The potential difference $\Delta V$ between the sides $(x=0)$ and $(x=L)$ is denoted by $\bar{\phi}$ and the potential difference between both graphene sheets is described by $\Delta \phi_{p}$. This value is nonlinearly dependent on the value of $\Delta V$ and is computed as a result of the FEM computation.

In what follows, we study the effects of both distance $d$ and energy barrier height $\Phi_{0}$ in Eq. (2). The Figure 9 presents the results of current density as a function of the electric field in the polymer $\bar{E}_{p}\left(\bar{E}_{p}=-\Delta \phi_{p} / d\right)$ and effective conductivity of the model computed as $\bar{k}=d \overline{J_{1}} / d \overline{E_{1}}$ as a function of the effective electric field $\bar{E}_{1}$, where $\bar{E}_{1}=-\Delta V / L$. First, the barrier height $\Phi_{0}$ is fixed to $0.17 \mathrm{eV}$ and the distance between the graphene sheets varies from 10 to $50 \AA$. We note that the current density increases with the electric field $\bar{E}_{p}$, while for a fixed $\bar{E}_{p}$, a smaller value of $d$ leads to a larger current density. 


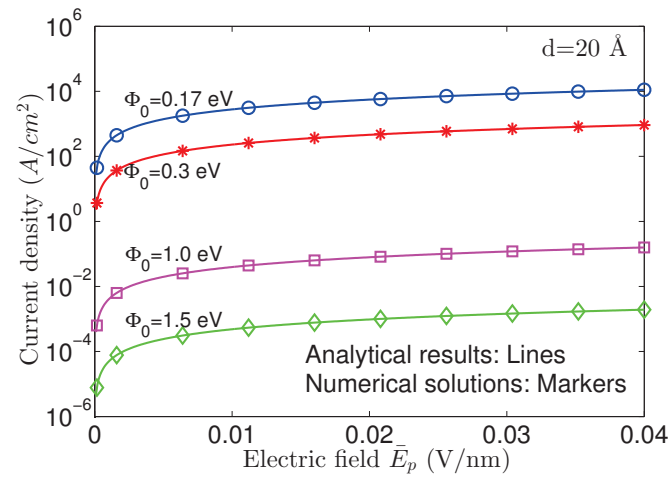

(a)

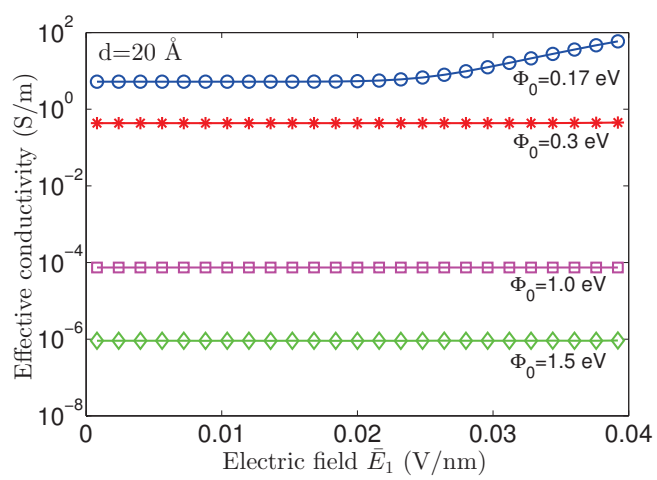

(b)

Figure 10: (a) Effective current density as a function of $\bar{E}_{p}$; (b) Effective electric conductivity as a function of $\bar{E}_{1}$ at different barrier height.

We compare in Fig. 9 (a) our numerical solution of current density and the analytical estimate provided by Eq. (2) for 1D tunnelling effect. We note a good agreement between the analytical and the numerical solution. Note that in the case $d=40 \AA$ and $d=50 \AA$, the current density increases sharply when $\bar{E}_{p}$ is over $0.03 \mathrm{~V} / \mathrm{nm}$ and finally reach the same value. This is because due to the tunnelling effect law, when the potential difference between the barrier is large enough, the current density tends to be independent on the barrier width $d$.

The Figure 9(b) indicates that the effective condutivity of this 2D model remains constant at low effective electric field $\bar{E}_{1}$ and increases sharply when $\bar{E}_{1}$ reaches a threshold, while for the same $\bar{E}_{1}$, the effective conductivity decreases with an increase of $d$.

To further investigate the influence of the barrier height $\Phi_{0}$, we fix the distance $d$ to $20 \AA$, and compute the current density and effective conductivity for the same structure as a function of $\bar{E}_{p}$ and $\bar{E}_{1}$ respectively. We observe from Fig. 10 that for lower barrier height $\Phi_{0}$, we obtain a higher current density and overall electric conductivity for the same electric field.

\subsection{RVE size analysis}

In this example, we analyze the convergence of the apparent (effective) conductivity of the composite as a function of the size of the RVE. Periodic boundary conditions are prescribed. For each size of the cubic domain defining the RVE, 30 realizations of random microstructures with the same 


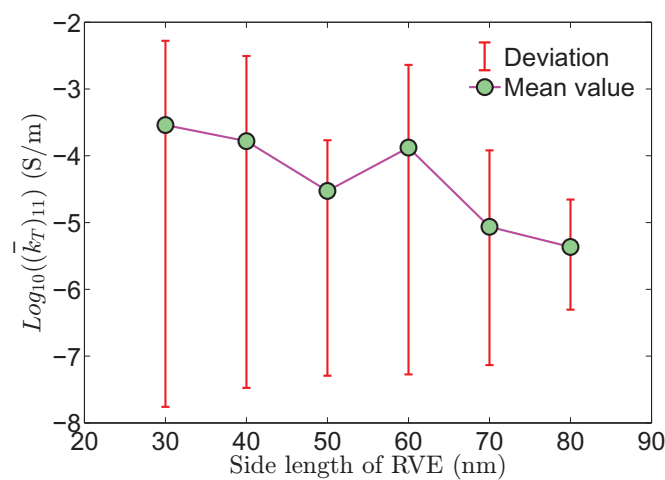

(a)

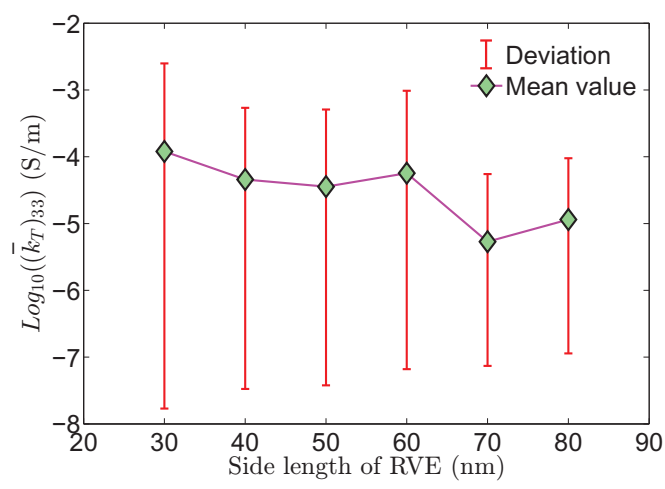

(c)

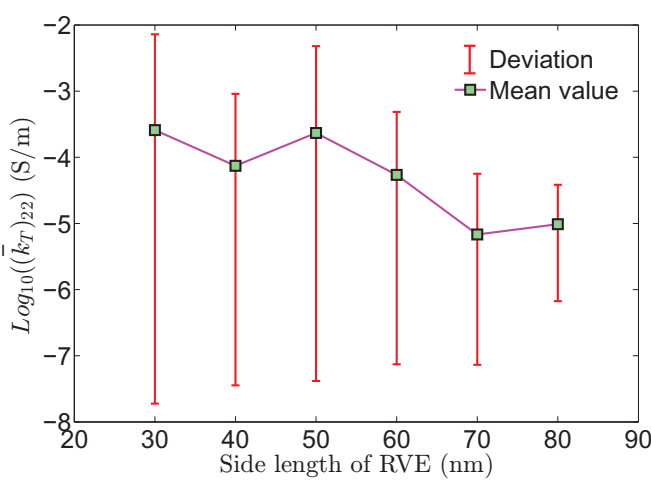

(b)

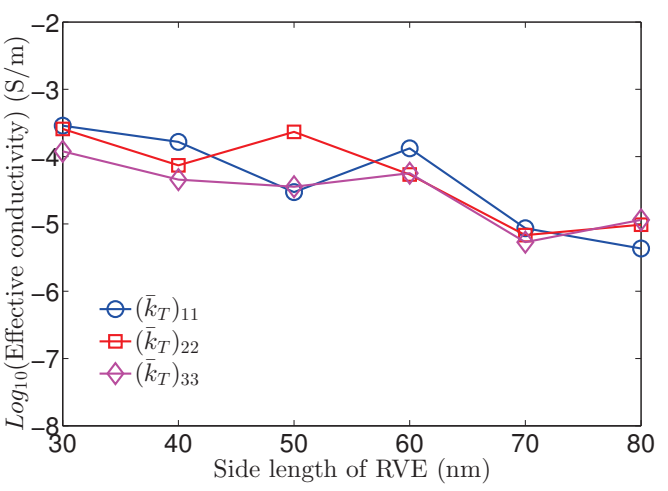

(d)

Figure 11: Effective conductivities tensor components as a function of RVE size, $\mathrm{f}=1.05$ vol\%, $\bar{E}=1.25 \times 10^{-3} \mathrm{~V} / \mathrm{nm}$. (a) $\left(\bar{k}_{T}\right)_{11}$ (b) $\left(\bar{k}_{T}\right)_{22}$ (c) $\left(\bar{k}_{T}\right)_{33}$ (d) Comparison of the three components.

graphene volume fraction $(1.05 \mathrm{vol} \%)$ and the same applied electric field $\left(1.25 \times 10^{-3} \mathrm{~V} / \mathrm{nm}\right)$, are computed to determine the apparent electric conductivity according to Eq. (21). Samples with side length ranging from 30 to $80 \mathrm{~nm}$ are analyzed. Graphene sheets are still modeled as square domains with dimensions $15 \times 15 \mathrm{~nm}^{2}$ and a thickness of $0.3 \mathrm{~nm}$, which is taken into account through the surface model in Eq. (13). Fig. 11(a-c) provides the effective conductivities tensor components $\left(\bar{k}_{T}\right)_{11},\left(\bar{k}_{T}\right)_{22},\left(\bar{k}_{T}\right)_{33}$ as a function of the domain size. It shows that the dispersion of results decreases when the size of the volume increases. In Fig. 11 (d), we can see that the mean values of electric conductivities along the three axes (x, y and $\mathrm{z}$ ) are close to each other in the case of random structures, leading to an isotropic behavior. 
The mean values converge when the side length of the RVE is roughly 80 $\mathrm{nm}$, which is the value we use in the next examples.

\subsection{Effective electric conductivity and percolation threshold}

Next, we use the proposed methodology to investigate the influence of graphene volume fraction on the effective electric conductivity of graphene/ polymer nanocomposites. The material of the matrix is PMMA polymer. The length of the cubic RVE is $80 \mathrm{~nm}$. The volume fraction is increased by accounting for the thickness of graphene sheets through (13) and by increasing the number of introduced graphene sheets in the domain. The graphene sheets are still square surfaces with a fixed size $15 \times 15 \mathrm{~nm}^{2}$ and a thickness of $0.3 \mathrm{~nm}$. The sheets are distributed randomly using a Markov-chain hard-plate algorithm (e.g. see [42] for example in 2D case of hard disk). The aspect ratio of graphene is 50. Several realizations of microstructures are presented in Fig. 12, where only the mesh of the graphene surfaces is shown.

The barrier height between graphene and PMMA is taken as $\Phi_{0}=0.17$ eV [43]. Periodic boundary conditions defined in Eq. (10) are prescribed. The problem being nonlinear, the effective conductivity is the incremental one as defined in (21), and depends on the intensity and history of the applied electric field. The examples are given for a fixed value $\bar{E}_{1}=0.0025 \mathrm{~V} / \mathrm{nm}$. The associated potential difference is $\Delta V=0.2 \mathrm{~V}$.

The numerical results are provided in Fig. 13 for PMMA/graphene nanocomposite with varying graphene volume fraction. Taking into account the tunnelling effect, the numerical values of $\left(\bar{k}_{T}\right)_{11},\left(\bar{k}_{T}\right)_{22}$ and $\left(\bar{k}_{T}\right)_{33}$ are plotted for each volume fraction as shown in Fig.13 (a-c), where the average values are obtained for 30 realizations. It indicates that the electric conductivity of the graphene-reinforced nanocomposites increases with the graphene volume fraction, and a sharp rise of conductivity can be noticed at about 0.8 vol\% where the mean value turns to be over $10^{-8} \mathrm{~S} / \mathrm{m}$. Meanwhile, we can see that the deviation of the conductivities corresponding to each 30 realizations whose graphene volume fraction is around $0.8 \mathrm{vol} \%$ is much larger.

For better comparison, we have superposed the mean values of $\left(\bar{k}_{T}\right)_{11}$, $\left(\bar{k}_{T}\right)_{22}$ and $\left(\bar{k}_{T}\right)_{33}$ in Fig. $13(\mathrm{~d})$ and also plotted the average value $\frac{1}{3}\left[\left(\bar{k}_{T}\right)_{11}+\right.$ $\left.\left(\bar{k}_{T}\right)_{22}+\left(\bar{k}_{T}\right)_{33}\right]$ when the tunnelling effect is neglected. It can be shown that the tunnelling effect is responsible for an increase in the apparent conductivity of several orders of magnitude, which is expected as the polymer matrix alone is almost isolating and that in our model, the graphene sheets are not 


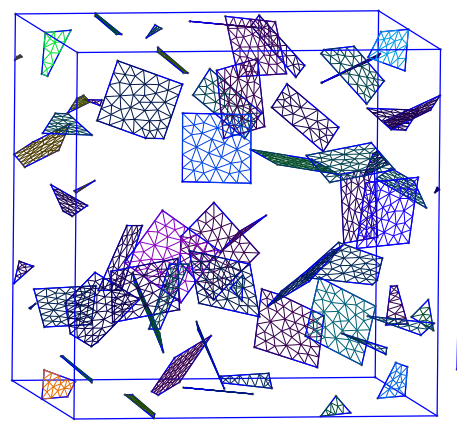

(a) $\mathrm{f}=0.40 \operatorname{vol} \%$

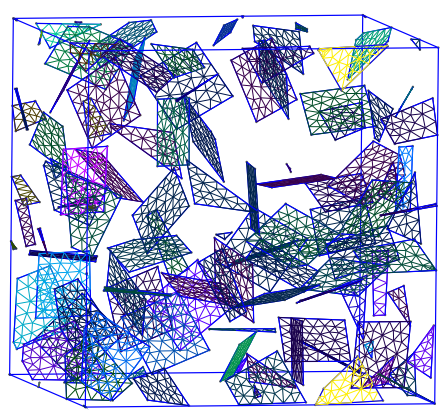

(d) $\mathrm{f}=0.79 \operatorname{vol} \%$

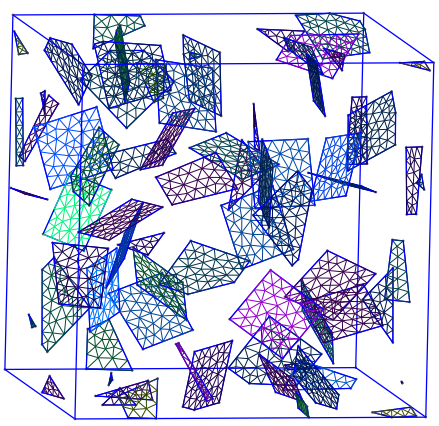

(b) $\mathrm{f}=0.53 \operatorname{vol} \%$

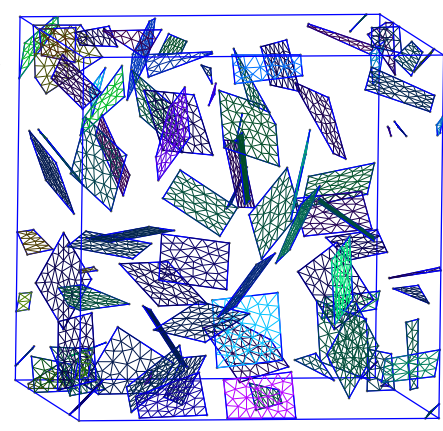

(c) $\mathrm{f}=0.66 \mathrm{vol} \%$

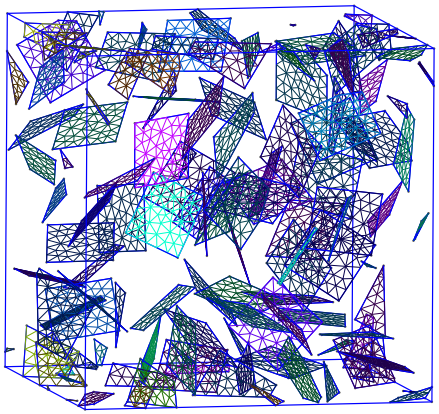

(e) $\mathrm{f}=0.92 \operatorname{vol} \%$

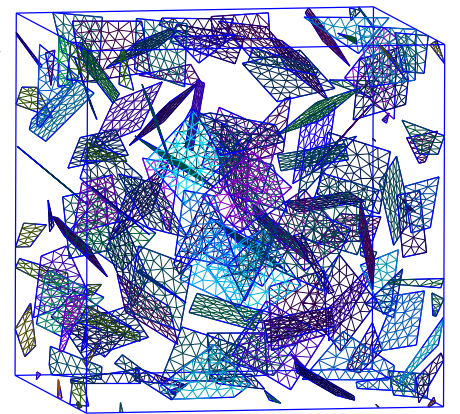

(f) $\mathrm{f}=1.05 \mathrm{vol} \%$

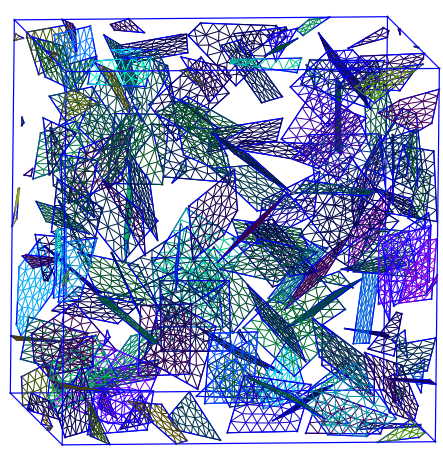

(g) $f=1.32 \operatorname{vol} \%$

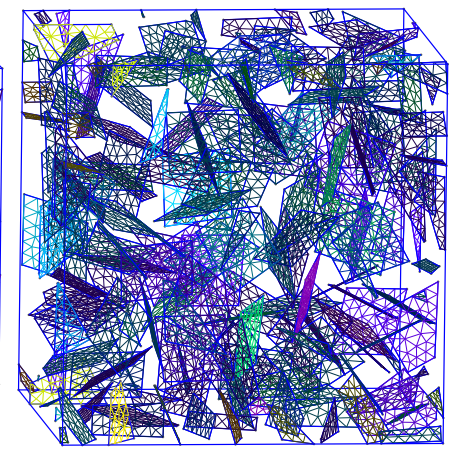

(h) $\mathrm{f}=1.58 \operatorname{vol} \%$

Figure 12: Realizations of microstructures for different graphene volume fractions. For visualization purpose, only the mesh of graphene sheets is depicted. 


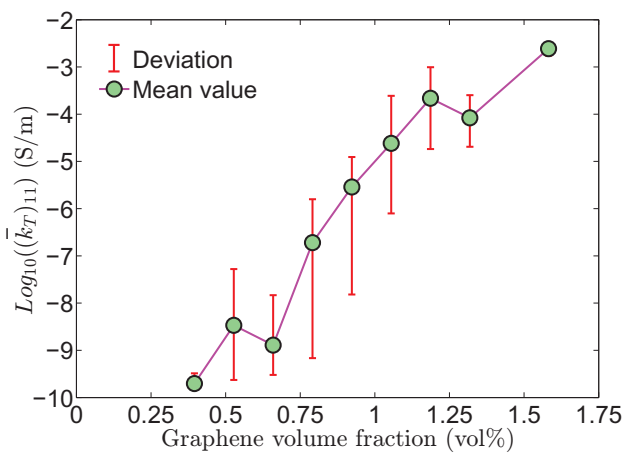

(a)

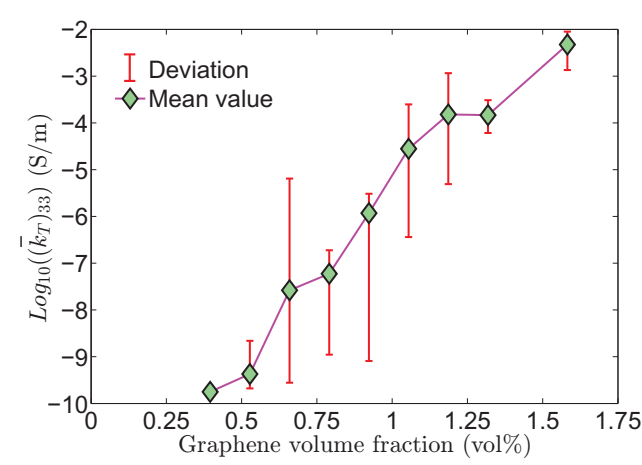

(c)

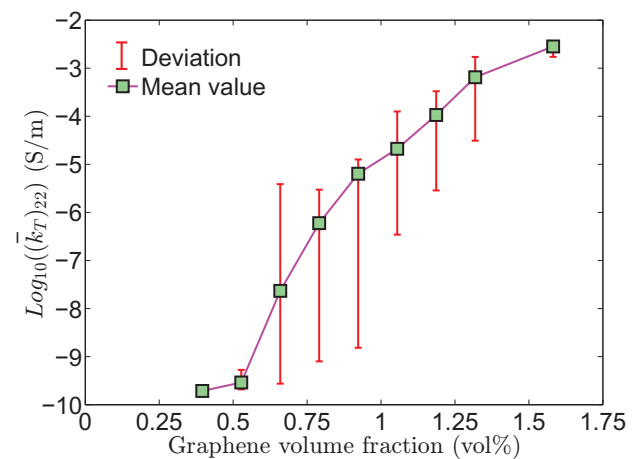

(b)

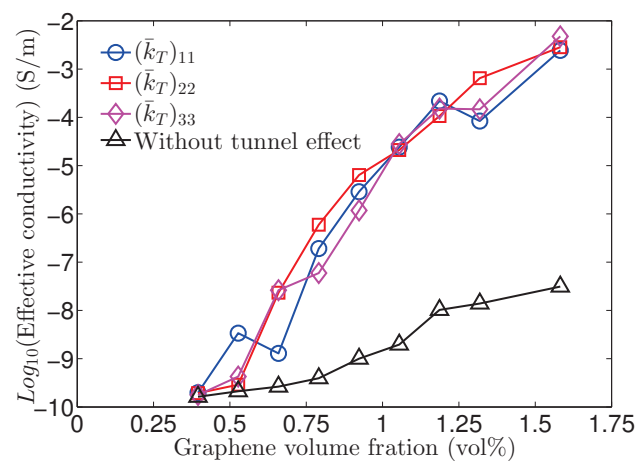

(d)

Figure 13: Effective conductivities tensor components as a function of the graphene volume fraction, $\bar{E}=1.25 \times 10^{-3} \mathrm{~V} / \mathrm{nm}, \Phi_{0}=0.17 \mathrm{eV}$. (a) $\left(\bar{k}_{T}\right)_{11}$ (b) $\left(\bar{k}_{T}\right)_{22}$ (c) $\left(\bar{k}_{T}\right)_{33}$ (d) Comparison with the case in which tunnelling effect is neglected.

in contact with each other. This insulator-to-conductor transition in composites made of a conductive filler and an insulating matrix is frequently described by percolation theory. And percolation threshold is the minimum filler content in the matrix which is characterised by a sharp rise of several orders of magnitude in conductivity due to the formation of conductive network. We estimate the percolation threshold around about 0.8 vol\% which indicates that a very small volume fraction of graphene can lead to a giant increase in the effective conductivity of the composite. These theoretical predictions are in good qualitative agreement with those available in [44].

We provide in Fig. 14 a comparison of the obtained results with and without tunnelling effect with available analytical bounds and estimates. To provide a fair comparison, numerical simulation are performed for infinitesi- 


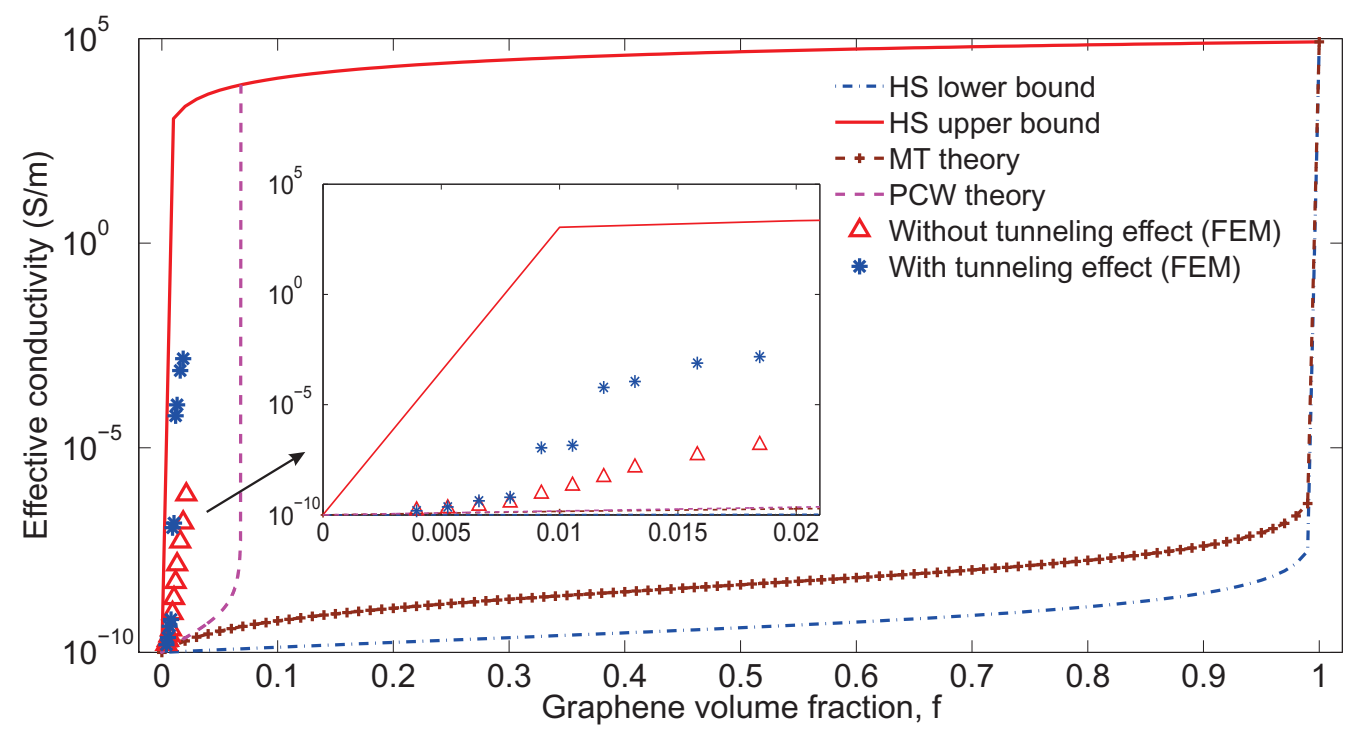

Figure 14: Comparisons between numerical solution of effective conductivity obtained by the present numerical framework and available analytical estimates in the linear regime.

mal applied electric fields, to remain in a linear regime. Analytical solutions are obtained by Hashin-Strikman (HS) bounds [45], Mori-Tanaka (MT) estimates [46, 47], and Ponte Castañeda Willis (PCW) estimates [48, 49], using ellisoids with high aspect ratio $(\gamma=50)$ to mimic the graphene platelets. Conductivities for polymer and graphene are taken as $k_{p}=1 \times 10^{-10} \mathrm{~S} / \mathrm{m}$ and $k_{g}=8.32 \times 10^{4} \mathrm{~S} / \mathrm{m}$, respectively. We can note a sharp increase of PCW estimate solution for graphene volume fraction $f=6.8$ vol\%. Such sharp increase has been noted also in [49]. Both numerical results with and without tunnelling effect remain within HS bounds, but show a sharp increase of effective conductivity for $0.8 \mathrm{vol} \%$. Then, the present framework seems to be more accurate to represent, even in the linear regime, the low percolation thresholds for graphene-reinforced nanocomposites observed as ranging from $0.1 \mathrm{vol} \%$ to $1 \mathrm{vol} \%[41,50,51]$.

In order to better understand the importance of tunnelling effect on the percolation behavior, the norm of the current density vector field in the polymer matrix of a random microstructure is presented in Fig. 15 for both with and without considering tunnelling effect respectively. The side length of the RVE cube is $40 \mathrm{~nm}$, and the graphene volume fraction is $1.05 \mathrm{vol} \%$. It is shown in Fig. 15 (a) that if tunnelling effect is neglected, the maximum 


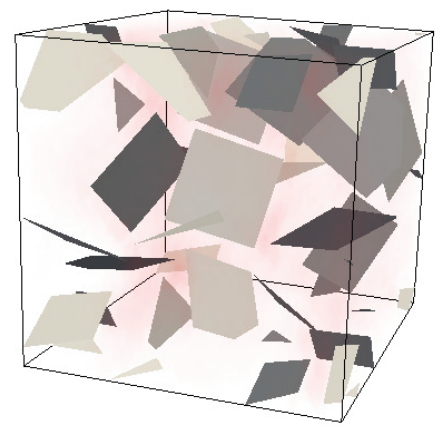

(a)

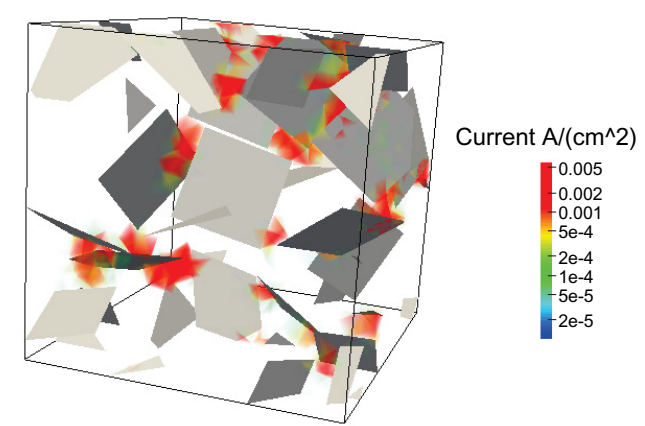

(b)

Figure 15: Norm of current density vector in the polymer matrix of microstructure for graphene volume fraction $f=1.05 \mathrm{vol} \%$, side length of RVE $l=40 \mathrm{~nm}$ : (a) without tunnelling effect; (b) considering tunnelling effect. The values below the minimum of scale bar are set to transparency. The ParaView post-treatment software was used [52].

current density in the matrix is about $10^{-9} \mathrm{~A} / \mathrm{cm}^{2}$, which indicates that the composite is insulating. While with the introduction of tunnelling effect, we can see the tunnelling current in the local matrix between graphene sheets in Fig. 15 (b), which is $10^{7}$ times larger than in Fig. 15 (a). In this case, a current percolation path can be observed due to the tunnelling current and leads to a insulator-conductor transition of the nanocomposite.

\section{Conclusion}

In this paper, we have proposed a numerical modeling framework for predicting the effective electric conductivity in polymer/graphene nanocomposites, taking into account tunnelling effect. A computational homogenization method has been developed, and the following features were introduced. First, a 3D tunnelling effect model of electric conduction for close graphene sheets has been proposed, by extending the 1D model to 3D and by defining an appropriate distance map function. Then, the related nonlinear equations of the electrical problem have been introduced. The nonlinear equations have been solved by a FEM technique, where the graphene sheets have been modeled using imperfect interfaces with high conductivity, which avoids meshing them along their thickness. Linearizations have been introduced and the effective conductivity has been evaluated numerically by appropriate definitions of effective quantities through homogenization. The method has been validated for benchmark problems and applied to RVE computations 
of polymer/grahene nanocomposites. Such procedure allows analyzing the effects of microstructural parameters on the effective electric conductivity. The predicted percolation thresholds are in good agreement with the available experimental data. From the numerical analysis, we can conclude that the tunnelling effect can explain qualitatively the experimentally observed nonlinear electric response in nanocomposites and a very low percolation thresholds.

\section{Aknowledgements}

JY thanks the financial support of Institut Universitaire de France (IUF). LU thanks the financial support of China Scholarship Council (CSC) for the Ph.D. thesis.

\section{References}

[1] Q. Li, Siddaramaiah, N. H. Kim, G.-H. Yoo, J. H. Lee, Positive temperature coefficient characteristic and structure of graphite nanofibers reinforced high density polyethylene/carbon black nanocomposites, Composites Part B: Engineering 40 (3) (2009) 218 - 224.

[2] Z. Spitalsky, D. Tasis, K. Papagelis, C. Galiotis, Carbon nanotube polymer composites: Chemistry, processing, mechanical and electrical properties, Progress in Polymer Science 35 (3) (2010) 357 - 401.

[3] O.-K. Park, T. Jeevananda, N. H. Kim, S. il Kim, J. H. Lee, Effects of surface modification on the dispersion and electrical conductivity of carbon nanotube/polyaniline composites, Scripta Materialia 60 (7) (2009) $551-554$.

[4] G. G. Tibbetts, M. L. Lake, K. L. Strong, B. P. Rice, A review of the fabrication and properties of vapor-grown carbon nanofiber/polymer composites, Composites Science and Technology 67 (2007) 1709 - 1718.

[5] W. Li, A. Dichiara, J. Bai, Carbon nanotube-graphene nanoplatelet hybrids as high-performance multifunctional reinforcements in epoxy composites, Composites Science and Technology 74 (2013) 221 - 227. 
[6] Y. Xu, Y. Wang, J. Liang, Y. Huang, Y. Ma, X. Wan, Y. Chen, A hybrid material of graphene and poly (3,4-ethyldioxythiophene) with high conductivity, flexibility, and transparency, Nano Research 2 (4) (2009) 343-348.

[7] J. Du, H. Cheng, The fabrication, properties, and uses of graphene/polymer composites, Macromolecular Chemistry and Physics 213 (2012) 1060-1077.

[8] S. J. Woltornist, J.-M. Y. Carrillo, T. O. Xu, A. V. Dobrynin, D. H. Adamson, Polymer/pristine graphene based composites: From emulsions to strong, electrically conducting foams, Macromolecules 48 (3) (2015) 687-693.

[9] M. Shtein, R. Nadiv, M. Buzaglo, K. Kahil, O. Regev, Thermally conductive graphene-polymer composites: Size, percolation, and synergy effects, Chemistry of Materials 27 (6) (2015) 2100-2106.

[10] H. Zhao, J. Bai, Highly sensitive piezo-resistive graphite nanoplateletcarbon nanotube hybrids/polydimethylsilicone composites with improved conductive network construction, ACS Applied Materials \& Interfaces 7 (18) (2015) 9652-9659.

[11] T. Wei, G. Luo, Z. Fan, C. Zheng, J. Yan, C. Yao, W. Li, C. Zhang, Preparation of graphene nanosheet/polymer composites using in situ reduction-extractive dispersion, Carbon 47 (9) (2009) 2296 - 2299.

[12] T. Wang, G. Liang, L. Yuan, A. Gu, Unique hybridized graphene and its high dielectric constant composites with enhanced frequency stability, low dielectric loss and percolation threshold, Carbon 77 (2014) 920 932.

[13] S. Vadukumpully, J. Paul, N. Mahanta, S. Valiyaveettil, Flexible conductive graphene/poly(vinyl chloride) composite thin films with high mechanical strength and thermal stability, Carbon 49 (1) (2011) 198 205.

[14] H. Pang, T. Chen, G. Zhang, B. Zeng, Z.-M. Li, An electrically conducting polymer/graphene composite with a very low percolation threshold, Materials Letters 64 (20) (2010) 2226 - 2229. 
[15] W. Bauhofer, J. Z. Kovacs, A review and analysis of electrical percolation in carbon nanotube polymer composites, Composites Science and Technology 69 (2009) 1486 - 1498.

[16] J. Wang, S. Yu, S. Luo, B. Chu, R. Sun, C.-P. Wong, Investigation of nonlinear i-v behavior of cnts filled polymer composites, Materials Science and Engineering: B 206 (2016) $55-60$.

[17] C. H. Hu, C. H. Liu, L. Z. Chen, Y. C. Peng, S. S. Fan, Resistancepressure sensitivity and a mechanism study of multiwall carbon nanotube networks/poly(dimethylsiloxane) composites, Applied Physics Letters 93 (3).

[18] J. Sandler, J. Kirk, I. Kinloch, M. Shaffer, A. Windle, Ultra-low electrical percolation threshold in carbon-nanotube-epoxy composites, Polymer 44 (19) (2003) 5893-5899, in Honour of Ian Ward's 75th Birthday.

[19] F. He, S. Lau, H. L. Chan, J. Fan, High dielectric permittivity and low percolation threshold in nanocomposites based on poly(vinylidene fluoride) and exfoliated graphite nanoplates, Advanced Materials 21 (6) (2009) 710-715.

[20] A. Allaoui, S. Hoa, M. Pugh, The electronic transport properties and microstructure of carbon nanofiber/epoxy composites, Composites Science and Technology 68 (2) (2008) 410 - 416.

[21] M. Martin-Gallego, M. Bernal, M. Hernandez, R. Verdejo, M. LopezManchado, Comparison of filler percolation and mechanical properties in graphene and carbon nanotubes filled epoxy nanocomposites, European Polymer Journal 49 (6) (2013) 1347 - 1353.

[22] X. Zeng, X. Xu, P. M. S. E. Kovalev, C. Baudot, N. Mathews, Y. Zhao, Characteristics of the electrical percolation in carbon nanotubes/polymer nanocomposites, The Journal of Physical Chemistry C 115 (44) (2011) 21685-21690.

[23] F. H. Gojny, M. H. Wichmann, B. Fiedler, I. A. Kinloch, W. Bauhofer, A. H. Windle, K. Schulte, Evaluation and identification of electrical and thermal conduction mechanisms in carbon nanotube/epoxy composites, Polymer 47 (6) (2006) 2036 - 2045. 
[24] J. Li, P. Ma, W. Chow, C. To, B. Tang, J.-K. Kim, Correlations between percolation threshold, dispersion state, and aspect ratio of carbon nanotubes, Advanced Functional Materials 17 (16) (2007) 3207-3215.

[25] F. Du, J. E. Fischer, K. I. Winey, Effect of nanotube alignment on percolation conductivity in carbon nanotube/polymer composites, Physical Review B 72 (12) (2005) 121404.

[26] N. Hu, Y. Karube, C. Yan, Z. Masuda, H. Fukunaga, Tunneling effect in a polymer/carbon nanotube nanocomposite strain sensor, Acta Materialia 56 (13) (2008) 2929 - 2936.

[27] W. S. Bao, S. A. Meguid, Z. H. Zhu, G. J. Weng, Tunneling resistance and its effect on the electrical conductivity of carbon nanotube nanocomposites, Journal of Applied Physics 111.

[28] J. G. Simmons, Electric tunnel effect between dissimilar electrodes separated by a thin insulating film, Journal of Applied Physics 34 (9) (1963) 2581-2590.

[29] A. Buldum, J. P. Lu, Contact resistance between carbon nanotubes, Physical Review B 63 (2001) 161403.

[30] Y. Wang, J. W. Shan, G. J. Weng, Percolation threshold and electrical conductivity of graphene-based nanocomposites with filler agglomeration and interfacial tunneling, Journal of Applied Physics 118 (6).

[31] J. Yvonnet, Q.-C. He, C. Toulemonde, Numerical modelling of the effective conductivities of composites with arbitrarily shaped inclusions and highly conducting interface, Composites Science and Technology 68 (13) (2008) $2818-2825$.

[32] F. T. Hamann C, Burghardt H, Electrical conduction mechanisms in solids, Vch Pub, 1988.

[33] C. Fu-Chien, A review on conduction mechanisms in dielectric films., Advances in Materials Science \& Engineering (2014) 1 - 18.

[34] J. G. Simmons, Conduction in thin dielectric films, Journal of Physics D: Applied Physics 4 (5) (1971) 613. 
[35] N. P. Maity, R. Maity, R. Thapa, S. Baishya, A tunneling current density model for ultra thin hfo2 high-k dielectric material based $\{$ MOS devices, Superlattices and Microstructures 95 (2016) $24-32$.

[36] S. Krishnan, E. Stefanakos, S. Bhansali, Effects of dielectric thickness and contact area on current-voltage characteristics of thin film metalinsulator-metal diodes, Thin Solid Films 516 (8) (2008) 2244 - 2250.

[37] S. Gu, Q.-C. He, Interfacial discontinuity relations for coupled multifield phenomena and their application to the modeling of thin interphases as imprefect interfaces, Journal of the Mechanics and Physics of Solids 59 (2011) 1413-1426.

[38] M. Geers, V. Kouznetsova, W. Brekelmans, Multi-scale computational homogenization: Trends and challenges, Journal of Computational and Applied Mathematics 7 (2010) 2175-2182.

[39] M. Geers, J. Yvonnet, Multiscale modeling of microstructure-property relations, MRS Bull. 41(08) (2016) 610-616.

[40] C. Geuzaine, J.-F. Remacle, Gmsh: A 3-d finite element mesh generator with built-in pre- and post-processing facilities, International Journal for Numerical Methods in Engineering 79 (11) (2009) 1309-1331.

[41] S. Stankovich, D. A. Dikin, G. H. B. Dommett, K. M. Kohlhaas1, E. J. Zimney1, E. A. Stach, R. D. Piner, S. T. Nguyen, R. S. Ruoff, Graphenebased composite materials, Nature 442 (7100) (2006) 282-286.

[42] W. Krauth, Statistical mechanics: algorithms and computations, Vol. 13, OUP Oxford, 2006.

[43] R. Rahman, P. Servati, Effects of inter-tube distance and alignment on tunnelling resistance and strain sensitivity of nanotube/polymer composite films, Nanotechnology 23 (5) (2012) 055703.

[44] E. Tkalya, M. Ghislandi, R. Otten, M. Lotya, A. Alekseev, P. van der Schoot, J. Coleman, G. de With, C. Koning, Experimental and theoretical study of the influence of the state of dispersion of graphene on the percolation threshold of conductive graphene/polystyrene nanocomposites, ACS Applied Materials \& Interfaces 6 (17) (2014) 15113-15121. 
[45] Z. Hashin, S. Shtrikman, A variational approach to the theory of the elastic behaviour of multiphase materials, Journal of the Mechanics and Physics of Solids 11 (2) (1963) 127-140.

[46] G. J. Weng, The theoretical connection between mori-tanaka's theory and the hashin-shtrikman-walpole bounds, International Journal of Engineering Science 28 (11) (1990) 1111-1120.

[47] G. J. Weng, Some elastic properties of reinforced solids, with special reference to isotropic ones containing spherical inclusions, International Journal of Engineering Science 22 (7) (1984) 845-856.

[48] P. P. Castañeda, J. R. Willis, The effect of spatial distribution on the effective behavior of composite materials and cracked media, Journal of the Mechanics and Physics of Solids 43 (12) (1995) 1919-1951.

[49] Y. Pan, G. Weng, S. Meguid, W. Bao, Z.-H. Zhu, A. Hamouda, Percolation threshold and electrical conductivity of a two-phase composite containing randomly oriented ellipsoidal inclusions, Journal of Applied Physics 110 (12) (2011) 123715.

[50] H.-B. Zhang, W.-G. Zheng, Q. Yan, Y. Yang, J.-W. Wang, Z.-H. Lu, G.-Y. Ji, Z.-Z. Yu, Electrically conductive polyethylene terephthalate/graphene nanocomposites prepared by melt compounding, Polymer 51 (5) (2010) 1191-1196.

[51] S. Y. Kim, Y. J. Noh, J. Yu, Prediction and experimental validation of electrical percolation by applying a modified micromechanics model considering multiple heterogeneous inclusions, Composites Science and Technology 106 (2015) 156-162.

[52] U. Ayachit, The ParaView Guide: A Parallel Visualization Application, Kitware, Inc., USA, 2015. 